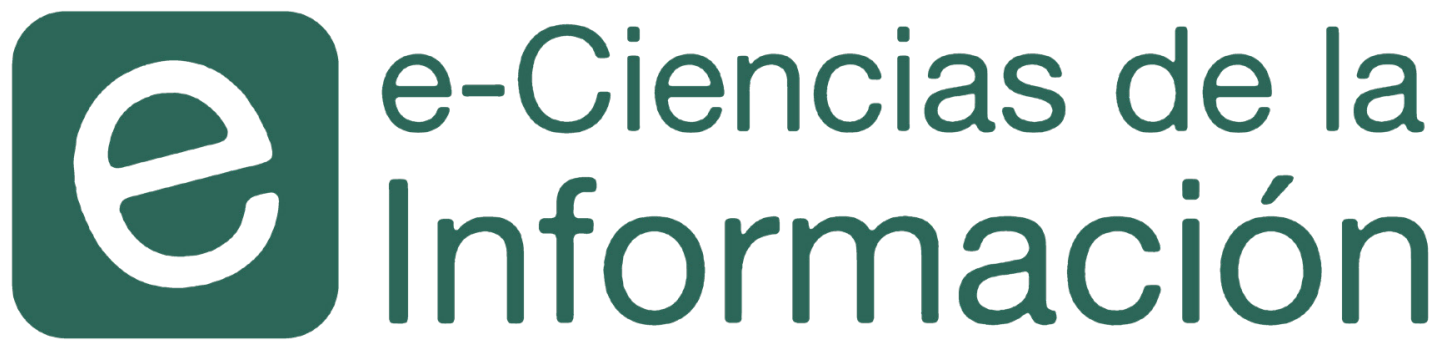

\title{
Las Bibliotecas públicas como promotoras del envejecimiento activo saludable
}

\section{Marcela Gil Calderón Lorena Chaves Salgado Roger Bonilla Carrión}

Recibido: 16/08/2019 | Corregido: 29/11/2019| Aceptado: 4/12/2019 DOI: $10.15517 /$ eci.v10i1.38667

e-Ciencias de la Información, volumen 10, número 1, Ene- Jun 2020

ISSN: 1649-4142

\section{다(1)(5)(2)}

\section{¿Cómo citar este artículo?}

Calderón Gil, M., Salgado Chaves, L. y Carrión Bonilla, R. (2020). Las Bibliotecas públicas como promotoras del envejecimiento activo saludable.e-Ciencias de la Información, 10(1).Doi: 10.15517/eci.v10i1.38667 


\title{
Las Bibliotecas públicas como promotoras del envejecimiento activo saludable
}

\author{
Public libraries as promoters of healthy and active \\ elderliness
}

\author{
Marcela Gil Calderón (10 ${ }^{1}$ \\ Lorena Chaves Salgado (1)2 \\ Roger Bonilla Carrión (10 ${ }^{3}$
}

\section{RESUMEN}

Las bibliotecas públicas son por excelencia, las organizaciones que reciben y trabajan con la población adulta mayor. Se presenta un proyecto desarrollado en la Escuela de Bibliotecología y Ciencias de la Información, de la Universidad de Costa Rica, cuyo objetivo general fue desarrollar estrategias lúdico-creativas, para promover la alfabetización informacional en salud en la población adulta y adulta mayor, desde las bibliotecas públicas. Es una investigación cualitativa, en que se aplicó la investigación acción. Los resultados alcanzados muestran que después de la aplicación de talleres lúdico-creativos, las personas adultas mayores aumentaron su conocimiento, interés y destreza en ellos. Así mismo se logró fomentar las relaciones intergeneracionales, pues los talleres fueron desarrollados por estudiantes de Trabajo Comunal Universitario.

\begin{abstract}
Palabras Clave: Acceso a la información, Servicios de información, Adultos mayores, Personas adultas mayores, Estilos de vida saludables, Bibliotecas públicas, Costa Rica
\end{abstract}

\section{ABSTRACT}

Public libraries are par excellence, the organizations that receive and work with the elderly population. The following is a project developed by the School of Library and Information Sciences of the University of Costa Rica whose general objective was to develop ludic and creative-based strategies to promote informational alphabetization and health in adults and the

\footnotetext{
${ }^{1}$ Universidad de Costa Rica, COSTA RICA. marcela.gil@ucr.ac.cr ORCID: https://orcid.org/0000-0002-1627$\underline{8782}$

${ }^{2}$ Universidad de Costa Rica, COSTA RICA._lorena.chaves@ucr.ac.cr ORCID: https://orcid.org/0000-00019169-7541

${ }^{3}$ Universidad de Costa Rica, COSTA RICA. roger.bonilla@ucr.ac.cr ORCID: https://orcid.org/0000-0002-8789$\underline{4494}$
} 
elderly population from the public libraries. It was a qualitative action-research investigation. Results evince an increase in interest, knowledge, and skills amongst the elderly after the implementation of the ludic and creative workshops. At the same time, it was feasible to promote intergenerational relations since the workshops were implemented by students as part of their Communal University Work.

Key Words: Information Access, information services, Elderly people, Healthy lifestyles, Public Libraries, Costa Rica

\section{Introducción}

Las bibliotecas públicas son los sitios por excelencia para brindar servicios a las personas adultas mayores. Se destacan como espacios de encuentro interactivo, en el que convergen diversos grupos de usuarios, cuya participación en actividades intergeneracionales, produce beneficios para mejorar su calidad de vida, entre los que se destacan por ejemplo, el fomento de redes sociales informales, grupos con intereses comunes y relaciones con distintos grupos etarios. Estas entidades pueden proveer a las personas adultas mayores una variedad de servicios; entre ellos sobresale la información educacional en el campo de la salud, que contribuye a un envejecimiento activo y saludable (Gil, 2016). Costa Rica mediante el Sistema Nacional de Biblotecas-SINABI, cuenta con 56 bibliotecas públicas, y además se suman a este grupo, las bibliotecas públicas de la Municipalidad de San José, lo que genera un potencial para que equipos interdisciplinarios promuevan actividades tendientes a favorecer a este conglomerado etario.

Como centros promotores socioculturales de las comunidades, las bibliotecas públicas, tanto a nivel nacional como internacional, pueden impulsar iniciativas en aras de ofrecer una mejor calidad de vida a las personas adultas mayores. Es por eso que la Ley Integral para la Persona Adulta Mayor-7935 establece que se debe promover el desarrollo integral, la cultura, la salud, entre otros aspectos. Así mismo en el reglamento a esta ley, se especifica en el artículo 19, inciso b que: "El Sistema Nacional de Bibliotecas creará un mecanismo para préstamo de libros y documentos a domicilio para las personas adultas mayores $y$, además, organizará actividades que permitan la interacción de estas personas con generaciones de niños, niñas y jóvenes" (Sistema Nacional de Bibliotecas, 2018, p. 20).

Este proyecto surge a raíz del intercambio de ideas y trabajo conjunto entre profesionales de la bibliotecología y ciencias de la información y gerontología de la Universidad de Costa Rica. Se planteó desde la visión gerontológica ${ }^{4}$, explorando las posibilidades de promoción del envejecimiento activo, al ofrecer a la población adulta mayor, de una forma innovadora, actividades lúdico-creativas ${ }^{5}$ cuya finalidad principal es que aprendan y se mantengan

\footnotetext{
${ }^{4}$ Gerontología: la ciencia que estudia el envejecimiento en todos sus aspectos, tanto biológicos como psicológicos o sociológicos, teniendo en cuenta, además, su evolución histórica y los factores referidos a la salud de la persona mayor (Millán Calenti, 2006, p.3)

${ }_{5}^{5}$ Metodología lúdico creativa: concepto de aprendizaje interactivo, donde la persona aprende mediante actividades de juego y creatividad, que pretende fomentar el pensamiento creativo, promover procesos de descubrimiento, experimentación, la imaginación y a la vez partir del análisis de la realidad sociocultural que le rodea (Murillo Rojas, 1998, p. 98)
} 
activos de una forma divertida y sana. La biblioteca además de brindar los servicios tradicionales que ya tiene establecidos, debe enfocar esfuerzos y orientar su línea de trabajo, para dar respuesta a las demandas muy particulares de los distintos grupos sociales que la circundan, abriendo espacios participativos de discusión, donde a las personas se les permita construir posibilidades, generar pensamientos nuevos y criticidad. En la actualidad una de las poblaciones que concurren con más regularidad y participa de las distintas actividades que brinda la biblioteca pública es la de las personas adultas mayores, esta población se caracteriza por ser muy activa y deseosa de aprender, ya que se beneficia por medio de las actividades que fomentan estilos de vida saludables que enriquecen las relaciones sociales al compartir con otras personas.

La Biblioteca destaca por su vocación de servicio y le brinda al adulto mayor un sitio donde encuentra bienestar por medio de estímulos diferentes que le animan a participar de propuestas nuevas y creativas que dan respuesta a la realidad social de este grupo con características tan demandantes y particulares. Tal como lo indica Gil (2016) "Las bibliotecas públicas son los espacios por excelencia para brindar servicios a las personas adultas mayores. Se enfatiza su potencial como un espacio de encuentro interactivo, en el que convergen diversos grupos de usuarios y usuarias, cuya participación en actividades con la población adulta mayor, genera beneficios para mejorar su calidad de vida" (p. 8).

Por lo tanto, las bibliotecas no deben concebirse sólo como depósitos para guardar colecciones de libros, sino más bien como espacios para propiciar actividades sociales, donde las personas mayores tengan la motivación para aprender y divertirse, lo que les ayudará a no sentirse solas ni excluidas, participando de un espacio de relación y convivencia que fomente un envejecimiento activo. Birren -citado por Rubio y Rubio (2011)- enfatiza que "Ios mayores que en la última etapa de la vida son relativamente aptos y tienen recursos necesarios para llevar a cabo actividades de forma consistente" ( $p$. $51)$.

La biblioteca debe considerarse como una red de apoyo que permita a la persona mayor erigir una gama de oportunidades que le brinden la posibilidad de crecer en su vida de forma integral, ofreciéndole actividades que respondan y contribuyan a un acompañamiento en el proceso del envejecimiento, que se prolonga toda nuestra vida y está condicionado al entorno social, económico en el que se vive.

\section{Referente teórico}

Los cambios de las condiciones de vida, el avance de la medicina y el conocimiento progresivo de la etiología de las enfermedades han dado curso a una reducción notable de la mortalidad, especialmente de la mortalidad temprana, y a un aumento del número de años de vida de la población (Comisión Económica para América Latina y el Caribe [CEPAL], 2012). Esta transición demográfica conduce a un envejecimiento poblacional, definido como el aumento de la proporción de personas adultas mayores ${ }^{6}$ con respecto al total de la población.

${ }^{6}$ Persona adulta mayor: Toda persona de sesenta y cinco años o más, según Ley 7935, Costa Rica. 
El envejecimiento poblacional tiene diferentes repercusiones desde la perspectiva de utilización de los servicios de salud. En Costa Rica, de acuerdo a la estructuración de nuestro Sistema de Salud, podemos referirlo desde el acceso al Primer Nivel de Atención hasta los servicios de mayor complejidad en los hospitales, siendo evidente en todos ellos el aumento de la demanda en forma paralela al aumento de personas adultas mayores. Estas necesidades de prestación de servicios de salud deben recibir una respuesta centrada en la persona, oportuna y sostenible a las necesidades en salud de las personas adultas mayores y acorde a la realidad social del país.

Los objetivos a cumplir en beneficio de las personas adultas mayores son, brindar una atención integral educando en salud, previniendo la enfermedad y la pérdida de funcionalidad, identificando a aquellas personas en situaciones de alto riesgo y realizar las intervenciones oportunas que les garantice una mayor probabilidad de mantener la salud y por ende la funcionalidad, prevenir el deterioro funcional y/o mental, y de esa forma asegurar una calidad de vida que dignifique el proceso de envejecimiento y aún de muerte de las personas mayores.

El envejecimiento de la población en el mundo es un fenómeno que atañe a la sociedad entera, pues afecta todos los ámbitos del quehacer del individuo y de la comunidad. Según lo señala el Fondo de Población de las Naciones Unidas (2012), el envejecimiento de la población es una de las tendencias más significativas en el siglo XXI. Tiene repercusiones importantes y de vastos alcances en todos los aspectos de la sociedad. A escala mundial, cada segundo dos personas cumplen 60 años, es decir, el total anual es de casi 58 millones de personas que llegan a los 60 años. Dado que actualmente una de cada nueve personas tiene 60 o más años de edad, y las proyecciones indican que la proporción será una de cada cinco personas hacia 2050, el envejecimiento de la población es un fenómeno que ya no puede ser ignorado (CEPAL, 2012, p.3).

Al ser un tema a escala mundial, se han generado una serie de acciones por parte de organismos internacionales, organismos no gubernamentales, y gobiernos de diversos países del orbe, para implementar políticas y programas que atiendan a la persona adulta mayor, de manera que aseguren su derecho a llevar una vida de calidad. Estas iniciativas, dependen en gran medida de la importancia y el interés mostrado por los tomadores de decisión, y de las condiciones políticas y socioeconómicas de cada país.

Una de las medidas que se promueve en la población es el envejecimiento activo, que la Organización Mundial de la Salud define como el "proceso de optimización de las oportunidades de salud, participación y seguridad que tiene como fin mejorar la calidad de vida de las personas a medida que envejecen" (Organización Mundial de la Salud, 2015, p. 248).

Los gobiernos y la sociedad en general deben garantizar el bienestar integral de las personas adultas mayores, procurándoles una vida digna y un desarrollo pleno. Instituciones como la Caja Costarricense del Seguro Social, la Universidad de Costa Rica y el Sistema Nacional de Bibliotecas, promueven actividades para apoyar a la población adulta mayor; no obstante, surge la necesidad de realizar un proyecto en que se realice una alianza estratégica interinstitucional que genere beneficios a largo plazo. En éste se busca incorporar las fortalezas de cada uno de los ejecutantes, para así establecer estrategias de prevención y promoción de la salud, con una perspectiva lúdico creativa, para involucrar no solo a la población adulta mayor, sino 
también a otros grupos etarios, con la intención de promover relaciones intergeneracionales en las cuales se beneficien todas las poblaciones, y a la vez, promover buenas prácticas de uso de la información en temas de promoción de la salud. Así mismo fue un proyecto interdisciplinario, en que participaron profesionales del área de la salud y de la bibliotecología, con lo que se incursionó en trabajos de corte social en el que aportaron distintas disciplinas.

El proyecto tiene como antecedente la realización de actividades en la Biblioteca Pública de Hatillo, durante el II Semestre de 2015, por parte de la Prof. Lorena Chaves, de la Escuela de Bibliotecología y Ciencias de la Información de la Universidad de Costa Rica. Se trabajó con un grupo de 20 personas adultas y adultas mayores de la comunidad de Hatillo no.2, ubicada en el distrito del mismo nombre, del cantón San José, diecisiete mujeres y tres hombres, cuyas edades oscilaron entre los 50 y los 90 años. Ellos asistieron a actividades recreativas tanto en la Biblioteca Pública de Hatillo como en la Casa del Adulto Mayor de la Municipalidad de San José, que está ubicada muy cerca de la Biblioteca. Con el fin de conocer su estado de salud, se les aplicó un cuestionario, cuyos resultados indicaron que entre las enfermedades que padecían en ese momento, predominaba en el grupo la hipertensión, seguida de problemas cardíacos y diabetes, además, todos manifestaron tener dificultades para leer y escribir. Este diagnóstico preliminar generó la posibilidad de proponer, establecer y evaluar estrategias para impulsar la alfabetización informacional en salud, particularmente en dos enfermedades crónicas no transmisibles, con alta incidencia en el país, como son la diabetes y la hipertensión arterial.

Es así como a inicios del 2016, tres docentes, dos de ellas docentes de la Escuela de Bibliotecología y Ciencias de la Información y otra docente de la Escuela de Enfermería de la Universidad de Costa Rica, proponen un Proyecto de Fondos Concursables ante la Vicerrectoría de Acción Social de dicha casa de estudios, titulado "Las Bibliotecas públicas como promotoras del envejecimiento activo saludable". Dicho proyecto fue aceptado e inició su ejecución a partir del 2016, como un Trabajo Comunal Universitario, definido según las Universidad de Costa Rica (UCR, 2018) como:

una actividad de acción social que vincula a grupos y comunidades vulnerables con la población estudiantil que cursa un plan de estudios en la Universidad de Costa Rica, cuyo propósito es contribuir con las transformaciones que la sociedad necesita. Esta experiencia interdisciplinaria es parte de la formación integral de la población estudiantil que responde a los principios y propósitos establecidos en el Estatuto Orgánico de la Universidad de Costa Rica, fundamentados en la ética y el respeto a los derechos humanos. EITCU es un requisito de graduación a nivel de pregrado y grado (p. 1).

Para llevar a cabo el proyecto, se establecieron los siguientes objetivos:

Objetivo General: Desarrollar estrategias para promover la alfabetización informacional en salud en la población adulta y adulta mayor, desde las bibliotecas públicas.

Objetivos específicos: Diseñar, aplicar y evaluar estrategias lúdico creativas para la promoción de la alfabetización informacional en salud en la población adulta y adulta mayor desde las bibliotecas públicas. 
Dicho proyecto se ejecutó durante dieciséis meses, de enero de 2016 a abril de 2017, en la Biblioteca Pública de Hatillo, ubicada en el Cantón central de San José, Costa Rica. Dicha Biblioteca, elegida por ser una institución cuya organización y proyección a la comunidad está muy consolidada, tiene las condiciones necesarias para trabajar con la población meta, su ubicación geográfica es de fácil acceso para los estudiantes y docentes que participarían en el proyecto. Aunado a esto está muy bien posicionada en la comunidad y tiene alianzas estratégicas con otras organizaciones que brindan servicios a la población adulta mayor, como la Municipalidad de San José y el Centro Diurno del Adulto Mayor de Hatillo.

Para la ejecución del proyecto se conformó un equipo interdisciplinario de trabajo, constituido inicialmente por docentes de la Universidad de Costa Rica, en el área de Bibliotecología y Enfermería con especialidad en Gerontología, así como el director de la Biblioteca Pública de Hatillo (Bibliotecólogo). Una vez iniciado el proyecto se integró otro docente de la Universidad de Costa Rica, funcionario de la Escuela de Bibliotecología y especialista en el área de la estadística, quien brindó asesoría puntual para cumplir con el objetivo de la evaluación de las estrategias lúdico creativas que se aplicaron, y la compañera docente del área de Enfermería, quien por motivos de fuerza mayor se retiró. Así mismo se contó con los estudiantes matriculados en el Trabajo Comunal Universitario TC-673, cuyas disciplinas fueron: Bibliotecología con énfasis en Ciencias de la Información, Bibliotecología con énfasis en Bibliotecas Educativas, Odontología, Oftalmología, Medicina y Promoción de la salud (25 estudiantes), cada uno de ellos aportó 300 horas de trabajo comunal universitario.

\section{Metodología}

La investigación que se llevó a cabo es de alcance exploratorio, que "se realizan cuando el objetivo es examinar un tema o problema de investigación poco estudiado, del cual se tienen muchas dudas o no se ha abordado antes" (Hernández, Fernández y Batista, 2010, p. 79).

\subsection{Enfoque}

Su enfoque es el cualitativo, definido como:

La investigación cualitativa se enfoca a comprender y profundizar los fenómenos, explorándolos desde la perspectiva de los participantes en un ambiente natural y en relación con el contexto. El enfoque cualitativo se selecciona cuando se busca comprender la perspectiva de los participantes (individuos o grupos pequeños de personas a los que se investigará) acerca de los fenómenos que los rodean, profundizar en sus experiencias, perspectivas, opiniones y significados, es decir, la forma en que los participantes perciben subjetivamente su realidad. También es recomendable seleccionar el enfoque cualitativo cuando el tema del estudio ha sido poco explorado, o no se ha hecho investigación al respecto en algún grupo social específico (Hernández et al., 2010, p. 364). 
Se aplicó la investigación-acción colaborativa, que en el campo educativo es muy utilizada, y es definida como "la que se realiza con la participación de personas provenientes de diferentes instituciones que deciden agruparse para reflexionar, analizar e investigar la práctica pedagógica, con el propósito de relacionar la investigación con procesos de innovación, de desarrollo y formación profesional con interés interpretativo" (Chaves, Díaz, García, Rojas y Solís, 2010, p. 25). En esta investigación cabe aclarar que se sustituyó la práctica pedagógica por la práctica bibliotecológica y de promoción de salud.

\subsection{Etapas de la investigación}

\subsubsection{Negociación de entrada}

Inicialmente se negoció el permiso para la realización del proyecto con la Dirección General de Bibliotecas Públicas y la Dirección de la Biblioteca Pública de Hatillo. Así mismo se hizo una convocatoria abierta a la comunidad de Hatillo, para invitar a adultos y adultos mayores a participar en el proyecto. Para esta convocatoria se invitó a la población adulta mayor que asistía a las actividades organizadas por la biblioteca pública, y se anunció en la celebración de la misa (Iglesia católica) de los domingos, en la Comunidad de Hatillo 8. Una vez conformados los grupos de adultos mayores, según las diversas actividades, se les explicó en forma detallada la finalidad y características del proyecto, sus objetivos, y se garantizó la confidencialidad de la información. Así mismo cada participante firmó un formulario de consentimiento informado, según lo establece el protocolo de la Universidad de Costa Rica.

\subsubsection{Descripción del escenario:}

El estudio se realizó en la Biblioteca Pública de Hatillo, que es una de las bibliotecas públicas más grandes del país, cuenta con instalaciones físicas amplias, en buen estado, incluye un auditorio, espacios para talleres y áreas verdes; así mismo, el edificio tiene un buen mantenimiento. Tanto estudiantes como docentes podían llegar al lugar, utilizando transporte público, con una parada de bus ubicada frente a en la Biblioteca Pública.

También trabajaron en el Centro Diurno de Hatillo, una casa de habitación adaptada para albergar durante el día a un grupo de adultos mayores, al estar cerca de la Biblioteca Pública, se logró establecer contacto con los encargados del lugar, y se trabajó también con estos adultos mayores, pues la Biblioteca Pública les brinda servicios de extensión bibliotecaria.

\subsection{Población del estudio}

Se contó con personas adultas mayores previamente identificadas, quienes llegaban a la Biblioteca Pública de Hatillo a participar en las actividades voluntariamente. Así mismo la Biblioteca Pública brinda servicios de extensión 
bibliotecaria7 al Centro Diurno de Hatillo, estos servicios contribuyen a salir del espacio físico habitual de la biblioteca y hacer uso de los recursos propios con que cuenta para desarrollar actividades que vayan en beneficio de una población meta, es este caso el grupo de adultos mayores que se sumó al estudio.

En las actividades de la Biblioteca Pública de Hatillo, específicamente con adultos mayores, se realizaron talleres desde mayo de 2016 a diciembre 2016. En algunos de los talleres participaron solo personas adultas mayores, en otras actividades participaron personas adultas mayores y personas adultas, para un aproximado de 50 personas adultas mayores y 15 personas de otras edades. Adicionalmente se trabajó con 20 adultos mayores del Centro Diurno de Hatillo, así que en total fueron 70 personas adultas mayores y 15 de otras edades (niños, jóvenes, adultos familiares de las personas adultas mayores que llegaron a las actividades). Para el análisis estadístico es importante señalar que se trabajó con el total de personas adultas mayores que participaron en cada taller analizado, y el número de participantes varió en cada taller.

Adicionalmente, los estudiantes que participaron en el Trabajo Comunal Universitario aportaron a la investigación desde su perspectiva de adultos jóvenes, trabajando con las personas adultas mayores, lo que enriqueció el estudio.

\subsection{Técnicas de recolección de datos}

Se utilizó inicialmente la observación participante que, Pretende recoger información en el medio natural donde se desarrolla; se fundamenta en la idea de que la convivencia personal del investigador con el grupo o institución investigada que se traduce en el acceso a todas las actividades del grupo, hace más fácil comprender las actuaciones de los sujetos, sus experiencias y procesos mentales (Colás y Buendía, 1994, p. 268).

Esta técnica permitió interactuar, cuestionar e intervenir en la situación observada. Adicionalmente el profesional en estadística en conjunto con las docentes de bibliotecología definieron los indicadores a evaluar, se diseñó el instrumento, se pidió a los estudiantes que lo aplicaran en algunas actividades (se seleccionaron de acuerdo al número de sesiones, ya que el instrumento se aplicó antes y después de la actividad), y se recolectó la información, para su posterior análisis.

Es importante señalar el papel relevante que tuvieron los estudiantes en la recolección de la información, pues ellos fueron los encargados de aplicar el instrumento.

Para la aplicación del instrumento mencionado, se siguió el enfoque de estudio de casos, una técnica muy utilizada en los estudios cualitativos que consiste en entrevistar a los participantes y que el encargado reporte en el instrumento sus impresiones (Stake, 1998).

\footnotetext{
7 Según Gómez Hernández (2007) "englobaríamos en la extensión bibliotecaria todas las actuaciones para tratar de eliminar o mitigar las barreras económicas, sociales o culturales que impiden o dificultan el acceso a la información de ciertos grupos de población".
} 


\section{Resultados}

Con respecto al indicador de impacto cualitativo, consistió en un cambio promedio antes-después de la estrategia lúdica. Dicho indicador consiste en medir antes y después de cada actividad de las estrategias lúdicas algunos aspectos básicos como conocimiento, interés y destrezas. A continuación, se obtiene un puntaje para cada participante antes y después de cada actividad, se obtiene el cambio (diferencia después-antes) a nivel individual y se obtiene un cambio promedio.

En dicho instrumento, se trabajó con las siguientes dimensiones:

Conocimiento, conjunto de información almacenada mediante la introspección (antes) o mediante la experiencia o el aprendizaje de la actividad de la estrategia lúdica (después).

Interés, afinidad o tendencia de los participantes hacia la actividad de la estrategia lúdica.

Destrezas, capacidad de los participantes para hacer bien, con facilidad y rapidez las diferentes actividades de la estrategia lúdica.

especto a los niveles, se definieron de la siguiente manera:

Nada, el participante no muestra ningún conocimiento, interés o destreza y hace las actividades con mucha dificultad o no las hace.

- Casi nada, el participante muestra escaso conocimiento, interés o destreza y hace las actividades con algún nivel de dificultad y las concluye con mucha ayuda.

- $\quad$ Algo, el participante muestra algunos conocimientos básicos, muestra interés y destreza y realiza las actividades con dificultad, y las concluye con ayuda.

- Bastante, el participante muestra muchos conocimientos, muestra mucho interés y destreza y realiza las actividades con facilidad, y las concluye sin ayuda.

- Mucho, el participante muestra muchos conocimientos, muestra mucho interés y destreza y realiza las actividades con facilidad y rapidez y hasta ayuda a los demás a concluirlas.

En forma complementaria se utilizó un formato de informe final del trabajo comunal universitario, diseñado para que los estudiantes expusieran de forma libre, su opinión sobre las tareas realizadas durante el Trabajo Comunal Universitario, y que permitió recolectar y sistematizar información valiosa para su posterior análisis. Adicional a esto, para documentar las reuniones de coordinación entre los participantes del proyecto, con el fin de dar seguimiento a lo planteado en las mismas, se elaboraron minutas o transcripción de grabaciones, en las cuales se hacía un resumen que incluyó lugar, fecha, hora, asistentes, temas y acuerdos.

\subsubsection{Plan de intervención}

Inicialmente, se les brindó a los estudiantes participantes en el Trabajo Comunal Universitario dos talleres de sensibilización para trabajar con adultos mayores, de manera que se familiarizaran con dicha población. Posteriormente, se realizaron reuniones generales e individuales, en que se coordinó la planificación, elaboración y ejecución de los talleres. En dichas reuniones se puntualizó en lo siguiente: 
1. Selección de temáticas.

2. Elaboración del taller.

3. Consentimiento informado.

4. Recolección de evidencias (fotos, entrevistas, cuestionarios).

5. Ejecución de los talleres.

6. Evaluación de talleres (aplicación de instrumento para evaluar las actividades).

7. Sesiones grupales para analizar lo aprendido y sistematizar experiencias.

Para elaborar la propuesta global de intervención, las docentes encargadas del Trabajo Comunal Universitario revisaron, corrigieron y avalaron cada taller propuesto por los estudiantes. Así mismo, para que fueran puestas en ejecución, se les pidió que cada taller tuviera los siguientes elementos:

1. Justificación del taller (antecedentes teóricos).

2. Propuesta del taller.

3. Coordinación con las docentes.

4. Coordinación con el Director de la Biblioteca Pública o la Directora del

Centro Diurno sobre temas como el espacio y duración de cada taller.

5. Aplicación del taller.

6. Que los grupos interdisciplinarios estuviesen conformados por dos o tres estudiantes.

7. Evaluación del taller.

8. Sistematización y análisis de la información.

Dichos talleres se aplicaron tanto en la Biblioteca Pública de Hatillo como en el Centro Diurno. Así mismo, se realizaron actividades específicas para el Festival del Adulto Mayor, que se llevó a cabo del 18 al 21 de octubre de 2016 en el marco del mes del adulto mayor. Dicho festival se llamó "Celebrando la vida". Durante esa semana se realizó una charla principal "Calidad de vida en la vejez", brindada por un motivador profesional.

Las áreas de trabajo para el diseño de los talleres fueron los siguientes:

1. Odontología: salud bucodental.

2. Medicina: diabetes, hipertensión.

3. Promoción de la salud: autocuidado, alimentación saludable, prevención y tratamiento de la hipertensión, baile, entre otras.

4. Oftalmología: cuidado de los ojos.

5. Literatura: taller de historias y café, taller de cuenta cuentos.

6. Cognición: juegos de mesa, juegos para promover la memoria, bingo, manualidades (reciclaje, navidad).

7. Alfabetización digital: talleres de computación, uso de teléfonos inteligentes.

\subsection{Procesamiento de análisis}

En la etapa de plan de intervención, se sistematizó la información generada a partir de los talleres, informes, observación participante, minutas de reuniones, resultados de la aplicación de instrumento. Como mencionan Hernández et al. (2010)

El análisis cualitativo implica organizar los datos recogidos, 
transcribirlos a texto cuando resulta necesario y codificarlos. La codificación tiene dos planos o niveles. Del primero, se generan unidades de significado y categorías. Del segundo, emergen temas y relaciones entre conceptos. Al final se produce teoría enraizada en los datos (p. 406).

Para realizar este análisis se hizo en varias etapas:

a. Las encargadas del proyecto, los funcionarios de la biblioteca pública y del centro diurno y los estudiantes se reunieron para reflexionar y analizar los resultados del mismo, en distintos momentos.

b. Análisis de los datos generados a partir de la información recolectada mediante el instrumento de medición del impacto cualitativo.

Se aplicó un cuestionario autoadministrado a una muestra de adultos mayores que participaban del TC-673, en la comunidad de Hatillo, San José, Costa Rica entre los meses de mayo a diciembre del año 2016. El cuestionario identificaba la actividad lúdica en donde participaba el entrevistado, el momento de la entrevista (antes o después de la actividad lúdica) y calculaba un puntaje para tres dimensiones: conocimiento, interés y destrezas. El puntaje para cada una de las dimensiones estaba basado en una escala ordinal de cinco puntos definido como: $\mathrm{Nada}=1$ punto, Casi nada $=2$ puntos, Algo $=3$ puntos, Bastante $=4$ puntos y Mucho $=5$ puntos. Así, por ejemplo, si el entrevistado participaba en el taller de reciclaje y fue entrevistado antes de dicha actividad, se le preguntaba " ¿Cuánto conocimiento tiene del taller de reciclaje?" y el entrevistado respondía "Casi nada", entonces la base de datos reportaba la siguiente información:

Actividad $=$ Taller de reciclaje

Momento $=$ Antes

Conocimiento $=2$

De forma similar se reportó el puntaje para las dimensiones de interés y destrezas. Una vez concluida la actividad lúdica, se volvió a aplicar el mismo cuestionario y en el momento de la entrevista se reportaba como "después".

Se calculó el promedio de los puntajes antes y los promedios después de cada actividad lúdica y sus respectivas desviaciones estándares y se realizó una prueba de hipótesis para la diferencia de los promedios con un $a=0.05$. La hipótesis de prueba es que los promedios de los puntajes antes y después de las actividades lúdicas son iguales. Se calculó el valor p para la prueba, en donde valores menores a $a=0.05$ hacen que se rechace la hipótesis nula.

Se calculó el cambio de los puntajes promedio $\Delta=$ después-antes, que es el indicador de impacto de las diferentes actividades lúdicas. El cambio de los puntajes $\Delta$ tiene valores positivos y negativos, si el valor $\Delta$ es positivo, indica que la actividad lúdica incrementó el conocimiento, el interés o las destrezas en los entrevistados. En caso que el valor $\Delta$ sea negativo, esto significa que la actividad lúdica disminuyó el conocimiento, el interés o las destrezas en los entrevistados. Si el valor $\Delta$ es cero, eso significa que no hubo ningún impacto. Se espera que los valores promedios del cambio de los puntajes $\Delta$ sea positivo para determinar un impacto de las actividades lúdicas. Se usó el paquete estadístico STATA para el procesamiento de la información (StataCorp, 2005). 
Se analizaron cinco talleres:

- Taller de navidad: Fomentó el uso de las destrezas de la motora fina, la estimulación cognitiva y la socialización. Por medio de la confección de manualidades navideñas como: monederos, un nacimiento, figuras de copos, de árboles y una campana. Para las personas adultas mayores es de suma importancia mantener activas sus capacidades físicas y cognitivas, por medio de la elaboración de manualidades que promueven entornos creativos y de entretenimiento.

-Taller de reciclaje: Creó conciencia sobre el reciclaje promoviendo un mejor uso de distintos materiales considerados de desecho y que dañan la naturaleza. Las personas adultas mayores recibieron principios de educación ambiental, lo cual les brindó la posibilidad de ampliar sus conocimientos sobre temas relacionados con el desarrollo sostenible y la conservación.

-Taller Aquileo J. Echeverría: Se analizaron sus obras y se hizo énfasis en el fomento del hábito de la lectura y la escritura como una práctica social y cultural incluyente que beneficia al adulto mayor brindándole conocimientos a nivel integral. Este taller versó sobre el escritor Aquileo J. Echeverría y sus aportes a la literatura costarricense, se fomentó el ejercicio de la lectura en voz alta, la escucha y la discusión de algunas de las "Concherías". Lo anterior interesó mucho a los participantes ya que hicieron remembranzas sobre costumbres de antaño, por lo tanto ejercitaron su memoria histórica.

-Taller de mandalas: Tuvo como objetivo fomentar la concentración cognitiva por medio de la técnica de colorear mandalas ya que dibujarlos o pintarlos representa una terapia. Se trabajó la expresión plástica para estimular aspectos físicos, psicológicos, emocionales y mentales en el adulto mayor.

-Taller de computadoras: El fin de este taller fue el de brindar los principios básicos de computación, incluyendo sistema operativo, equipo, y programas de cómputo del área de la ofimática. La alfabetización digital es una necesidad básica de la población adulta mayor, que fortalece su independencia y aprendizaje en el ambiente digital.

Con el fin de validar la información, se realizó la de triangulación de datos, que "consiste en contrastar e interpretar los datos, considerando diversas fuentes, técnicas de recolección, actores y referentes teóricos." (Chaves et al., 2010, p. 85).

Con base en el análisis de la información, se generaron las siguientes categorías de análisis (Ver Tabla 1): 
TABLA 1

Categorías de análisis

\begin{tabular}{|c|c|c|}
\hline $\begin{array}{l}\text { CATEGORÍA DE } \\
\text { ANÁLISIS }\end{array}$ & DEFINICIÓN CONCEPTUAL & SUBCATEGORÍAS \\
\hline $\begin{array}{l}\text { Alfabetización } \\
\text { informacional en salud }\end{array}$ & $\begin{array}{l}\text { La alfabetización en salud (AS) es la capacidad de los } \\
\text { individuos de acceder y utilizar información para tomar } \\
\text { decisiones sobre su salud (Montesi, 2017, p.1) }\end{array}$ & \\
\hline Estilos de vida saludable & $\begin{array}{l}\text { Promover la buena salud y los comportamientos } \\
\text { saludables a todas las edades para prevenir o retrasar la } \\
\text { aparición de enfermedades crónicas (OMS, 2012, p.7) }\end{array}$ & $\begin{array}{l}\text { Cognición } \\
\text { Salud }\end{array}$ \\
\hline Alfabetización digital & $\begin{array}{l}\text { Representa la habilidad del individuo para realizar } \\
\text { tareas efectivamente en un ambiente digital (Federación } \\
\text { de Enseñanza de CC.OO. De Andalucía, 2011, p.1) }\end{array}$ & $\begin{array}{l}\text { Computación } \\
\text { Dispositivos móviles }\end{array}$ \\
\hline $\begin{array}{l}\text { Estrategias lúdico- } \\
\text { creativas }\end{array}$ & $\begin{array}{l}\text { Se entiende como una forma de vivir e interactuar con } \\
\text { los otros en espacios cotidianos que produzcan disfrute, } \\
\text { gozo y alegría por medio de actividades simbólicas e } \\
\text { imaginarias tales como el juego, el arte, la literatura, el } \\
\text { movimiento y el sentido del humor, el afecto entre otros" } \\
\text { (Unfried, 2009, p. 10) }\end{array}$ & Actividades \\
\hline $\begin{array}{l}\text { Relaciones } \\
\text { intergeneracionales }\end{array}$ & $\begin{array}{l}\text { Las relaciones que se establecen entre individuos } \\
\text { de diferentes cohortes de edad o "Generaciones" } \\
\text { (Envejecimiento activo, 2019) }\end{array}$ & \\
\hline $\begin{array}{l}\text { Aprendizaje a lo largo de } \\
\text { la vida }\end{array}$ & $\begin{array}{l}\text { Se funda en la integración del aprendizaje y la vida, y } \\
\text { comprende actividades de aprendizaje para personas de } \\
\text { todas las edades, en todos los contextos (familia, escuela, } \\
\text { comunidad, lugar de trabajo, etc.) y mediante diversas } \\
\text { modalidades (educación formal, no formal e informal), } \\
\text { que en conjunto responden a una amplia gama de } \\
\text { necesidades y exigencias relativas al aprendizaje. } \\
\text { (Organización de las Naciones Unidas para la Educación, } \\
\text { la Ciencia y la Cultura [UNESCO], 2019) }\end{array}$ & $\begin{array}{l}\text { Adulto mayor } \\
\text { Estudiantes del Trabajo } \\
\text { Comunal Universitario }\end{array}$ \\
\hline
\end{tabular}

Fuente: Elaboración propia, 2017

\section{Resultados}

Las categorías de análisis definidas en la Tabla 1 permiten agrupar con claridad los temas analizados. En primer término, con respecto a la Alfabetización informacional en salud y estilos de vida saludable se les brindó a las personas adultas mayores talleres sobre alimentación saludable, higiene bucal y corporal, enfermedades crónicas, autocuidado y salud ocular. Se profundizó en dichos temas, de acuerdo a las necesidades y condiciones del grupo. Sin embargo, en esta categoría no se logró recibir retroalimentación por parte de la población participante con respecto a su opinión. No obstante, se aprecia en la Tabla 3 que, en el Taller de alimentación saludable, los participantes incrementaron su interés, conocimiento y destrezas después de recibirlo.

En relación con las estrategias lúdico-creativas, se procuró que todas las actividades estimularan el gozo, la creatividad y el disfrute por medio del juego, la literatura, el arte. Esto les permitió explayar sus emociones, tal como 
lo manifestaron los participantes en los siguientes comentarios, "me gustan mucho, mellenan de alegría"(Mujer de 75 años),"estoy muy contento"(Hombre de 97 años), "estuvo muy bonito el taller porque nos entretuvimos" (Mujer de 65 años). Así mismo se promovió el trabajo colaborativo, la socialización y el afecto, según lo indica esta mujer de 79 años "muy importante porque hay una comunión de uno y los otros".

El entretenimiento estuvo presente en todas las actividades, ya que uno de los fines fue propiciar un ambiente armónico, tranquilo y de disfrute, "para mí fue muy importante, me ha ayudado en mi mente, en mis manos, en mi conocimiento de los colores, en la depresión. Porque me distrae, me motiva y me alegra. Bendiciones por esta actividad ya que no tenía la destreza" (Mujer de 74 años). "Me gusta para entretenerme” (Hombre de 91 años).

Las manualidades y mandalas permitieron incentivar a los participantes en el uso de destrezas de la motora fina y la estimulación cognitiva. En estas sesiones de trabajo fue muy interesante notar que la mayoría de los participantes no habían vuelto a pintar desde su niñez, y algunos manifestaron que nunca lo habían hecho, por lo que en un inicio representó para algunos de ellos un reto. Al inicio de cada actividad se les dio a los participantes una justificación de los beneficios de las prácticas que se iban a efectuar y cómo esto iba a contribuir en el fortalecimiento del área cognitiva. Los participantes expresaron comentarios como: "me gustó pintar, y traté de no salirme de las líneas" (Mujer de 98 años), "me entretiene mucho y me levanta la autoestima, puedo combinar los colores (Mujer de 75 años), "a quien interese señores, por este medio les comunico que los trabajitos que se hicieron en días anteriores, relacionados a las magdalas (sic), fueron muy bonitos, aunque yo no tenía conocimiento, pero era la primera vez que lo hacía, mil gracias" (Hombre de 69 años). "Es excelente, tranquilizar la mente. Me gusta mucho, muy creativo y me gustaría volver a recibir esto, ya que a esta edad necesito mucho de estas cosas." (Mujer de 77 años).

El aprendizaje a lo largo de la vida, se evidenció en muchas de las actividades realizadas. Los participantes tomaron consciencia de que, a pesar de la edad, pueden adquirir conocimiento y poner en práctica lo aprendido. Se evidencia esto en los siguientes comentarios: "es muy importante mantener mi mente ocupada, este trabajo no es fácil, pero se puede seguir intentándolo a pesar de los años, se puede aprender" (Mujer de 74 años). "Aprender es un arte de la vida porque la vida es arte. Se tiene que aprender de todo lo que sea posible de acuerdo a la capacidad y el gusto" (Hombre de 63 años). "Todo lo puedo aprender, es bueno porque la vida se trata de eso. Solo hice la escuela nunca me fomentaron leer libros de escritores nacionales" (Hombre de 63 años). "Muy buen taller, aprendí a hacer las diferencias y analizar conceptos a ver más allá de lo que superficialmente uno valora. Recordé cosas olvidadas y pude profundizar" (Mujer de 74 años).

En lo que respecta a las relaciones intergeneracionales, las personas adultas mayores trabajaron con estudiantes del Trabajo Comunal Universitario, entre los que estaban adultos jóvenes y adultos. En este aspecto se logró obtener información de los participantes y los instructores. Respecto a los instructores, se advierte un cambio positivo en su concepción de la persona adulta mayor, como alguien que es capaz de trabajar, de reflexionar acerca de diferentes situaciones, que enfrenta necesidades reales en su vida y que es un grupo que necesita apoyo, compromiso, paciencia y solidaridad. Para conocer esto se les consultó específicamente ¿qué habían aprendido después de terminar el Trabajo comunal universitario? Esto se refleja en comentarios como los 
siguientes: "me despertó la conciencia social de la ayuda que necesitan los grupos poblacionales vulnerables", "aprendí a valorar los aportes de la población adulta mayor a la sociedad costarricense", "resalto el impacto del trabajo que como profesionales realizamos los bibliotecólogos con las distintas poblaciones que asisten a la biblioteca pública".

Con respecto al grupo de adultos mayores, su interacción con los jóvenes les brindó la posibilidad de interiorizar que son capaces de seguir aportando conocimiento a la sociedad, y que ocupan un lugar muy importante en el entorno que los rodea, lo que fortalece su autoestima. Para ejemplificar esto, "mi agradecimiento a mis profesores, por sus grandes dotes de gente, interés, paciencia y consideración demostrados en su gran destreza para enseñarme todo aquello que aprendí" (Hombre de 57 años).

Se buscó también realizar la medición del impacto cualitativo de cinco talleres, a continuación se analizan los resultados.

La Tabla 2 presenta el promedio de puntajes antes y después según las tres dimensiones. Existe un aumento significativo en el conocimiento $(p=0.000)$, interés $(p=0.000)$ y destrezas $(p=0.000)$ de los participantes hacia las actividades lúdicas.

TABLA 2

Promedio de puntajes* antes y después según dimensiones. Costa Rica, 2016.

\begin{tabular}{|c|c|c|c|c|c|}
\hline \multirow[b]{2}{*}{ DIMENSIONES } & \multicolumn{2}{|c|}{ ANTES } & \multicolumn{2}{|c|}{ DESPUÉS } & \multirow[b]{2}{*}{ P-VALUE } \\
\hline & PROMEDIO & D.E. & PROMEDIO & D.E. & \\
\hline Conocimiento & 2.5 & (1.3) & 4.1 & $(0.9)$ & $p=0.000$ \\
\hline Interés & 4.1 & (0.9) & 4.6 & (0.6) & $p=0.000$ \\
\hline Destrezas & 3.4 & $(1.2)$ & 4.1 & $(0.9)$ & $p=0.000$ \\
\hline
\end{tabular}

*Nivel de conocimiento: $\mathrm{Nada}=1$, casi nada $=2$, algo $=3$, bastante $=4$, mucho $=5$.

Fuente: Elaboración propia, 2016

La Tabla 3 presenta el promedio de puntajes antes y después según dimensiones y actividad lúdica. La Figura 1, Figura 2 y Figura 3 presentan el cambio de los puntajes $\Delta$ de las tres dimensiones según actividad lúdica. El taller de mascarada es la actividad lúdica que más cambios significativos presenta en la dimensión de conocimiento $(\Delta=2.5)$, el taller de reciclaje en el interés $(\Delta=1.2)$ y el uso de computadoras en el de destrezas ( $\Delta=$ 1.2). El impacto de las actividades lúdicas del programa de alfabetización informacional fue positivo. 
TABLA 3

Promedio de puntajes* antes y después según dimensiones y actividad lúdica. Costa Rica, 2016.

\begin{tabular}{|c|c|c|c|c|c|}
\hline & \multicolumn{2}{|c|}{ ANTES } & \multicolumn{2}{|c|}{ DESPUÉS } & \multirow[b]{2}{*}{ p-value } \\
\hline $\begin{array}{l}\text { Dimensiones/ act. } \\
\text { lúdica }\end{array}$ & Promedio & D.E. & Promedio & D.E. & \\
\hline \multicolumn{6}{|l|}{ Taller de mandalas } \\
\hline Conocimiento & 2.2 & $(1.5)$ & 3.9 & $(1.0)$ & $p=0.000$ \\
\hline Interés & 4.3 & $(1.0)$ & 4.3 & $(0.8)$ & $p=0.916$ \\
\hline Destrezas & 4.0 & $(0.9)$ & 4.3 & $(0.8)$ & $p=0.310$ \\
\hline \multicolumn{6}{|l|}{ Taller A. Echeverría } \\
\hline Conocimiento & 2.1 & $(0.9)$ & 4.1 & $(0.8)$ & $p=0.000$ \\
\hline Interés & 4.2 & $(0.7)$ & 4.5 & $(0.7)$ & $p=0.211$ \\
\hline Destrezas & 3.0 & (1.7) & 4.2 & $(0.9)$ & $p=0.052$ \\
\hline \multicolumn{6}{|l|}{ Taller de Navidad } \\
\hline Conocimiento & 3.3 & (1.4) & 4.5 & $(0.7)$ & $p=0.022$ \\
\hline Interés & 4.4 & $(0.8)$ & 4.5 & $(0.5)$ & $p=0.538$ \\
\hline Destrezas & 3.8 & $(1.2)$ & 4.4 & $(0.7)$ & $p=0.195$ \\
\hline \multicolumn{6}{|l|}{ Taller de reciclaje } \\
\hline Conocimiento & 2.2 & $(1.5)$ & 3.3 & $(1.5)$ & $p=0.147$ \\
\hline Interés & 3.5 & $(1.1)$ & 4.7 & $(0.5)$ & $p=0.018$ \\
\hline Destrezas & 2.5 & (1.3) & 3.1 & $(1.2)$ & $p=0.316$ \\
\hline \multicolumn{6}{|l|}{ Uso de computadoras } \\
\hline Conocimiento & 3.0 & $(0.6)$ & 4.3 & $(0.5)$ & $p=0.002$ \\
\hline Interés & 4.2 & $(0.8)$ & 4.8 & $(0.5)$ & $p=0.098$ \\
\hline Destrezas & 2.8 & $(0.4)$ & 4.0 & $(0.8)$ & $p=0.014$ \\
\hline \multicolumn{6}{|l|}{ Taller mascarada } \\
\hline Conocimiento & 2.0 & (1.4) & 4.5 & $(0.7)$ & $p=0.155$ \\
\hline Interés & 5.0 & $(0.0)$ & 4.5 & (0.7) & $p=0.423$ \\
\hline Destrezas & 4.5 & (0.7) & 4.5 & $(0.7)$ & $p=1.000$ \\
\hline \multicolumn{6}{|l|}{ Alimentación saludable } \\
\hline Conocimiento & 3.1 & $(1.0)$ & 4.1 & (0.7) & $p=0.023$ \\
\hline Interés & 3.8 & (0.7) & 4.6 & (0.5) & $p=0.020$ \\
\hline Destrezas & 3.2 & (0.4) & 4.0 & (0.6) & $p=0.004$ \\
\hline
\end{tabular}

*Nivel de conocimiento: $\mathrm{Nada}=1$, casi nada $=2$, algo $=3$, bastante $=4$, mucho $=5$. 
FIGURA 1

Cambio de los puntajes promedio en el conocimiento según actividad lúdica.

Costa Rica, 2016.

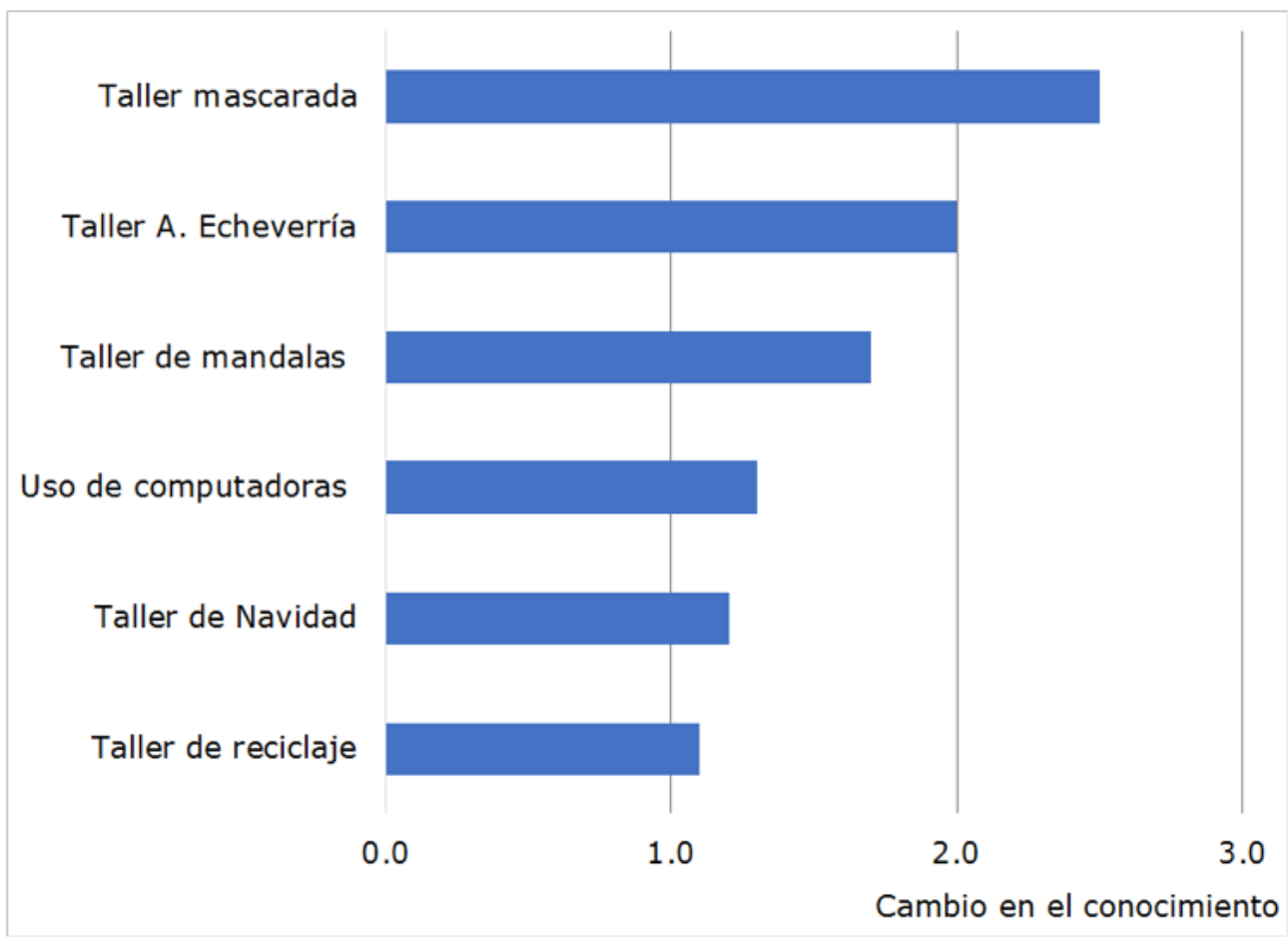

Fuente: Elaboración propia, 2016

FIGURA 2

Cambio de los puntajes promedio en el interés según actividad lúdica. Costa Rica, 2016.

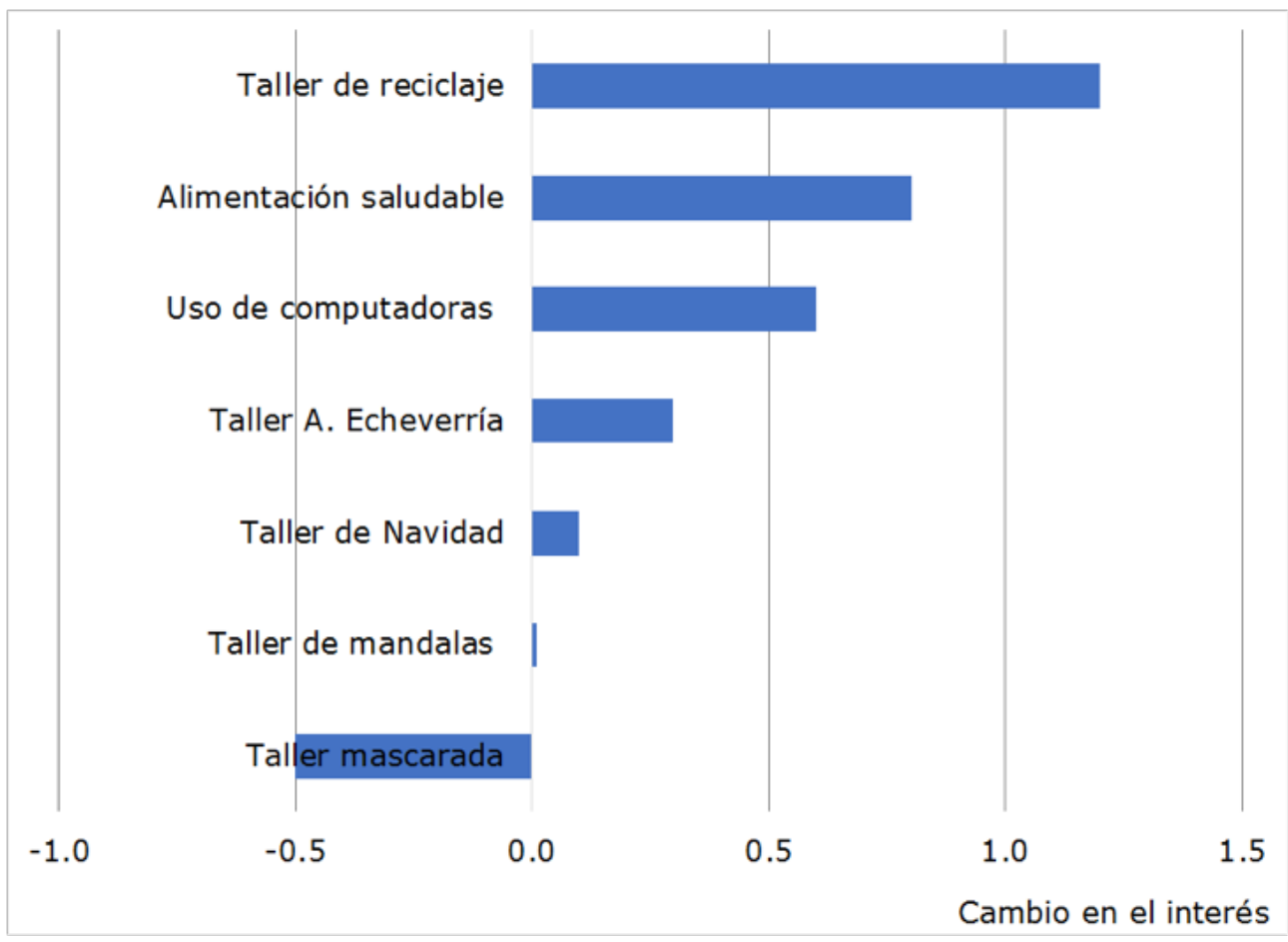


FIGURA 3

Cambio de los puntajes promedio en las destrezas según actividad lúdica. Costa Rica, 2016.

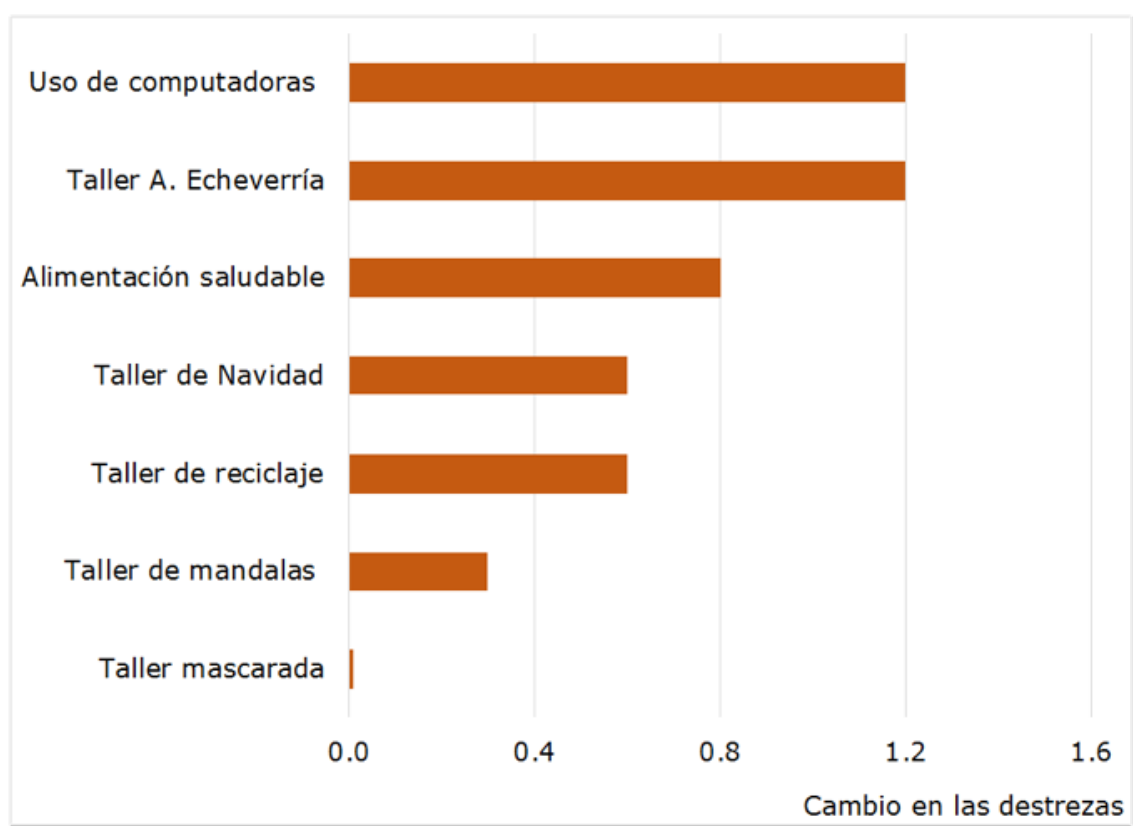

Fuente: Elaboración propia, 2016

\section{Conclusiones}

Se desprende del estudio que la generación de programas dirigidos a las personas adultas mayores es necesario, por cuanto el reto de los profesionales en Bibliotecología y Ciencias de la Información, con respecto a la atención de la persona adulta mayor en Costa Rica, sugiere el desafío de poner en práctica y desarrollar nuevos proyectos para que este grupo etario se integre de forma más justa y con equidad a la sociedad. Por este motivo surge la oportunidad de considerar a la biblioteca pública como un centro de alfabetización en salud, informacional, digital; que puede y debe contribuir por medio de servicios y recursos de interés a brindar atención al colectivo con una oferta de servicios gratuitos y abiertos.

Concurrir a la Biblioteca Pública debe significar para el adulto mayor obtener conocimientos que le sirvan y pueda poner en práctica para solventar sus necesidades de información, participando de actividades intelectuales, de entretenimiento y ocio. Ellos asisten voluntariamente, en algunos casos a pesar de tener limitaciones físicas, pero aún asíllegan, porque la biblioteca constituye un lugar de acogida, donde van al encuentro de multiplicidad de aprendizajes, de ideas y de sensaciones nuevas. Es el lugar para hacer amigos y para compartir entre pares y con personas de otros grupos etarios, es donde se divierten con distintas actividades por ejemplo talleres, bailes y encuentros literarios. La biblioteca se convierte en su segunda casa donde las vivencias que experimentan les permiten construir posibilidades y generar nuevos pensamientos ante el mundo que los rodea, es decir, se constata que el aprendizaje es a lo largo de toda la vida. 
Por medio de la investigación realizada se logró evidenciar el impacto que tuvo poner en práctica el proyecto por medio del Trabajo Comunal Universitario$\mathrm{TCU}$, como proceso académico interdisciplinario y colaborativo, mediante el cual se realizaron las actividades planeadas para las personas adultas mayores. Los estudiantes trabajaron con mucha dedicación y respeto hacia el grupo poniendo en práctica la tolerancia, el compromiso y la creatividad para impactar a la población cumpliendo metas reales. Este TCU permitió a los estudiantes tener un acercamiento al campo profesional, reconociendo las debilidades y fortalezas que se deben enfrentar, las cuales constituyen un constante fortalecimiento de las relaciones intergeneracionales.

Los servicios de extensión bibliotecaria hacia el Centro Diurno de Hatillo permitieron desarrollar parte de los objetivos propuestos en el proyecto. El grupo de adultos mayores beneficiado en este caso cumplía con características distintas que el grupo que asistió a la biblioteca pública. Aun así, ambos participaron de forma activa y gozaron de los espacios que las distintas actividades ofrecían.

En respuesta a las categorías de análisis planteadas se hace palpable la necesidad de poner en práctica con las personas adultas mayores talleres con estrategias creativas que estén enfocados a ofrecerles conocimientos y técnicas, de tal manera que ellos experimenten cambios positivos apoyados en un aprendizaje participativo, para que de forma integral obtengan herramientas específicas que puedan poner en práctica para atender sus necesidades, por medio de la resolución de objetivos específicos derivados de sus intereses. Otro aspecto importante que se evidenció es la necesidad de crear recursos educativos y materiales didácticos con características propias para la puesta en práctica de las actividades educativas llevadas a cabo en los talleres que cumplan con los objetivos del desarrollo de los contenidos propuestos.

Con el adulto mayor el aprendizaje es un proceso sensible y complejo que requiere de tiempo porque muchas veces hay que revertir viejos conceptos, costumbres y actitudes. Por eso la importancia de que las personas que intervienen en la ejecución de las actividades estén bien informadas sobre las características de la población y hayan tenido un proceso de sensibilización para acercarse a ellos de forma ética y tolerante

Con las categorías expuestas se hace manifiesta la importancia de trabajar con el adulto mayor tanto el área física como cognitiva, apoyando estos procesos con miras al bienestar y mejoramiento de la vida de este colectivo, promoviendo un envejecimiento activo saludable, etapa de la vida en donde se producen cambios de forma gradual tanto a nivel físico como psicológico y también social, lo que implica que esta población en aumento cada vez más en nuestro país requiera de una atención perseverante como un objetivo a conseguir por medio de una vocación constante, teniendo como marco de acción la biblioteca pública con actividades que les puedan atraer a visitarla.

\section{Agradecimientos}

Los autores agradecen al Lic. Oscar Rodríguez, Director de la Biblioteca Pública de Hatillo y a todo el personal de la misma, quienes contribuyeron desinteresadamente en este Proyecto. Así mismo a la Dra. Marlen Agüero Arce, que impulsó el proyecto y por motivos laborales no pudo seguir en el mismo. 
A la Licda. Priscilla Vargas del Centro Diurno de Hatillo, a los estudiantes del TC673 y a las personas adultas mayores que con tanto entusiasmo participaron en el proyecto, porque sin ellos no hubiera sido posible la investigación.

\section{Referencias}

Chaves, L., Díaz, M., García, J., Rojas, G. y Solís, N. (2010). Investigaciónacción colaborativa: Un encuentro con el quehacer cotidiano del centro educativo para su transformación. San José, Costa Rica: INIE.

Colás M. y Buendía, L. (1994). Investigación Educativa. Sevilla: ALFAR.

Comisión Económica para América Latina y el Caribe. (2012). Observatorio demográfico. Santiago, Chile: Autor.

Envejecimiento Activo (2019). Intergeneracionalidad. Recuperado de: $\quad$ https://envejecimientoactivo.wordpress.com/definicionintergeneracionalidad/

Fondo de Población de las Naciones Unidas. (2012) Envejecimiento en el Siglo XXI: una celebración y un desafío [Resumen Educativo]. Estados Unidos, Nueva York. Recuperado de https://www.unfpa.org/sites/default/ files/pub-pdf/Ageing\%20Report\%20Executive\%20Summary\%20 SPANISH\%20Final 0.pdf

Gil, M. (2016). La persona adulta mayor desde la biblioteca: una revisión sistemática. e-Ciencias de la Información, 6(1). Doi: http://dx.doi. org/10.15517/eci.v6i1.21833

Gómez Hernández, J. A. (2007). Biblioteca e integración: de la extensión bibliotecaria a los procesos de inclusión social y digital. En Gimeno, J., López, P., Morillo, M.J. De volcanes Ilena. Biblioteca y compromiso social (pp. 343-371). Gijón, España:Trea.

Hernández, R., Fernández, C. y Baptista, M.P. (2010). Metodología de la investigación (5 de.). México: McGraw-Hill. 
Ley integral para la persona adulta mayor 7935 y su Reglamento (1999). San José, Costa Rica, CONAPAM.

Millán Calenti, J.C. (2006). Principios de geriatría y gerontología. Madrid: McGraw-Hill Interamericana.

Montesi, M. (2017). Alfabetización en salud:revisión narrativa e interdisciplinar de la literatura publicada en biomedicina y en biblioteconomía y documentación. Revista Cubana de Información en Ciencias de la Salud, 28(3). Recuperado de http://scielo.sld.cu/scielo.php?script=sci arttext\&pid=S2307-21132017000300007\&lng=es\&tlng=es.

Murillo Rojas, M. (1998). La metodología lúdico creativa: una alternativa de educación no formal. Recuperado de www.waece.org/biblioteca/pdfs/ d098.pdf

Organización de las Naciones Unidas para la Educación, la Ciencia y la Cultura. (2019). Intergeneracionalidad. Recuperado de www.unesco. org/new/es/santiago/education/lifelong-learning/

Rubio, R. y Rubio, L (2011). El envejecimiento con éxito en el adulto mayor: algunos indicadores europeos. En La salud de los adultos mayores: una visión compartida (p. 51). Washington, Estados Unidos: Organización Panamericana de la Salud.

Sistema Nacional de Bibliotecas. (2018). Portal del SINABI: actividades. Recuperado de http://www.sinabi.go.cr/consultaactividades.aspx

Stake, R. (1998). Investigación con estudio de casos. Madrid, España: Ediciones Morata.

StataCorp. (2005). Stata Statistical Software: Release 9. College Station, TX: StataCorp LP.

Universidad de Costa Rica. (2018). Reglamento de trabajo comunal universitario. Recuperado de http://www.cu.ucr.ac.cr/no cache/ normativa/orden-alfabetico/Control/Normative.html 


\section{e-Ciencias de la Información}

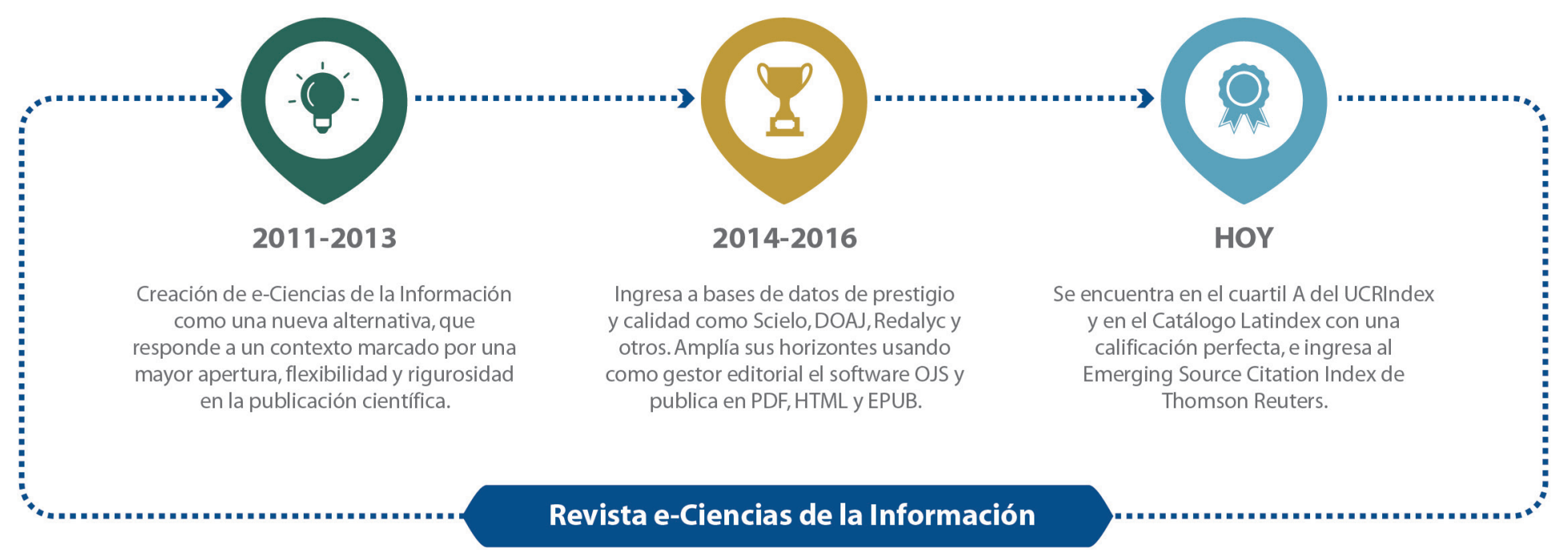

¿Dónde se encuentra idexada e-Ciencias de la Información?

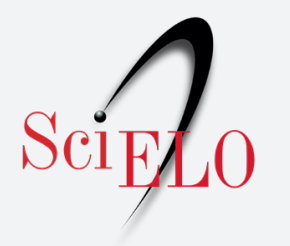

\section{latindex 12 clarivate Analytics}

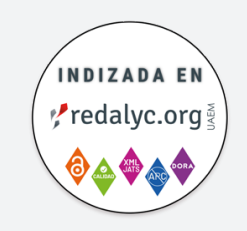

Para más información ingrese a nuestra lista completa de indexadores

¿Desea publicar su trabajo?

Ingrese aquí

O escríbanos a la siguiente dirección revista.ebci@ucr.ac.cr 